Petrich, Dominik; Dang, Thao; Breuel, Gabi; Stiller, Christoph: Assessing

Map-Based Maneuver Hypotheses using Probabilistic Methods and Evidence Theory.

In: Proc. IEEE Int. Conf. Intelligent Transportation Systems, pp. 995-1002, 2014

\title{
Assessing Map-Based Maneuver Hypotheses using Probabilistic Methods and Evidence Theory
}

\author{
Dominik Petrich $^{1}$, Thao Dang ${ }^{1}$, Gabi Breuel ${ }^{1}$ and Christoph Stiller ${ }^{2}$
}

\begin{abstract}
The prediction of the behavior of other traffic participants and the generation of respective motion hypotheses is a key capability of advanced driver assistance systems and autonomous vehicles. Motion prediction is a difficult task since it has to deal with the uncertainty within the environmental perception and the ambiguity of a traffic scene. For this reason we propose a two-layer situation analysis concept in this paper. This includes an associative and predictive situation model which combines probabilistic object hypotheses with a stochastic model of the road network in a curve coordinate system. Utilizing this description, we formulate various hypotheses regarding the evolvement of the situation using an Extended Kalman Filter supported by the Intelligent Driver Model. Furthermore, we introduce an evidence theory based situation interpretation to assess the several behavior hypotheses as well as to determine the inherent uncertainty. Especially in ambiguous situations, the ability to determine the imprecision by the difference of belief and plausibility of a certain hypothesis provides suitable information for an appropriate reaction. Both layers of the proposed situation analysis are not relying on training data and so it is not limited to previous known traffic scenarios. Finally, the capability of the concept is demonstrated by evaluating 157 maneuvers, recorded at an urban intersection.
\end{abstract}

\section{INTRODUCTION}

Due to the number of dynamic interacting traffic participants and maneuver possibilities, urban intersections are one of the most challenging traffic scenarios. To assist the driver for a safe passage through intersections, advanced driver assistance systems (ADAS) require detailed knowledge of the vehicle surrounding. This holds true not only for detecting the environment but also for getting additional information about the current situation the vehicle is in [1]. Especially for systems with an increasing degree of automation and a rising number of sensors the situation analysis (SitA) forms the link between the perception and assist function layer. Thus, SitA can be seen as the process of reaching situation awareness by providing an adequate description of the current traffic situation, the prediction of the future evolvement and the criticality assessment [2].

The key challenges in this domain deal with the unavoidable uncertainty within the environment perception and the ambiguities of the traffic scene. Following [1], [3], [4] and [5], the inherent uncertainty of a situation has to be distinguished into at least two general categories:

1) Inductive: Uncertainty as a state of mind

\footnotetext{
${ }^{1}$ The authors are with the Daimler AG, RD/drivingautomation, Boeblingen, Germany \{dominik.petrich, thao.dang, gabi.breuel\}edaimler.com

${ }^{2}$ The author is with the Institute of Measurement and Control Systems, Karlsruher Institute of Technology, Germany stillerekit.edu
}

2) Empirical: Uncertainty as a physical property of information

The first meaning of uncertainties consists in the vagueness of the detected objects in the vehicle environment in the sense of "is this object a car or a bicycle?". The second meaning results from the sensors which deliver imperfect data, often limited to basic features like position, orientation and velocity [6]. An additional uncertainty in meaning of the first category occur by deriving situation awareness from limited knowledge about the actual behavior of the traffic participants. This means that the reasons of decision making for individual drivers, like their plans or the parts of the scene they have seen, remain unknown. In summary, the situation analysis has to deal with the uncertainty of sense (data driven, empirical) and further with the uncertainty in behavior (goal driven, inductive).

\section{A. Related Work}

In the field of situation understanding several work has been performed to anticipate behavior of traffic participants in urban scenarios. To deal with the inherent uncertainties the most common approaches include (dynamic) Bayesian networks (BN) ([6], [7]), Hidden Markov Models (HMM) and Support Vector Machines (SVM) [8]. To regard multiple hypothesis the approaches [9] and [1] use Multi-hypothesis Tracker (MHT) respectively Fuzzy Sets with decisions trees. Particularly the results of (D)BN and SVM methods depend here strongly on the quantity and quality of the training data. All approaches have in common, that they differ not between inductive and empirical uncertainty in the resulting analysis. To meet these requirements, the SitA layer in this work is realized by a two-step process in which each kind of uncertainty is treated separately.

The remainder of this paper is organized as follows. Section II gives an overview to the complete system architecture and characterizes the input and output of the proposed SitA layer. A brief introduction to the theoretical background for a transformation in a stochastic curved coordinate system is described in Section III. In Section IV we formulate an associative situation model (SitM) based on the information of the environmental perception. Beside the combination of uncertain object hypothesis with the stochastic description of the local road network we generate various maneuver hypotheses regarding the evolvement of the current situation. Subsequently in Section V, the second layer of the SitA is introduced. Here, the defined maneuver hypotheses of the traffic participants are evaluated and associated with the intention of the system vehicle. This corresponds to a 


\begin{tabular}{|c|c|c|}
\hline ADAS Function & $\begin{array}{l}\text { Maneuver } \\
\text { Planning }\end{array}$ & $\begin{array}{l}\text { Driver Information } \\
\text { and Vehicle Control }\end{array}$ \\
\hline \multicolumn{3}{|r|}{$\overline{1}$} \\
\hline \multicolumn{2}{|c|}{ Situation Awareness } & Intention to Act \\
\hline \multicolumn{2}{|c|}{1} & $l$ \\
\hline Situation Analysis & $\begin{array}{l}\text { Situation Model } \\
\text { (SitM) }\end{array}$ & $\begin{array}{c}\text { Situation Interpretation } \\
\text { (Sitl) }\end{array}$ \\
\hline \multicolumn{2}{|c|}{1} & $\overline{1}$ \\
\hline \multicolumn{2}{|c|}{ Environment Perception } & Plausibility Check \\
\hline \multicolumn{2}{|l|}{1} & $I$ \\
\hline Data Acquisition & $\begin{array}{c}\text { Measured Data } \\
\text { (Object Hypotheses) }\end{array}$ & $\begin{array}{l}\text { Stored Data } \\
\text { (Digital Map) }\end{array}$ \\
\hline
\end{tabular}

Fig. 1. General system structure with different layers

subjective assessment of the situation, thus we call it situation interpretation (SitI). Finally, in Section VI the proposed concept is evaluated by real data recorded at an urban intersection.

\section{System OVERVIEW}

The main objective of the SitA is to provide the function layer of the ADAS a state of knowledge (called situation awareness) in a way it is able to make a decision about the next steps of the ego vehicle. Figure 1 illustrates an overview of the entire system structure. In this section we give a brief introduction to the applied architecture, except for the situation analysis, which will be discussed in section IV and V.

\section{A. Data Acquisition}

The data acquisition layer provides the two fundamental inputs for our SitA module by recognizing the traffic environment and evaluating map data. In contrary to the often locally stored map data, the measurements of the traffic scene can either be provided by the sensor system of the ego vehicle or communicated by Car2X. As shown in the research initiative Ko-FAS [10], possible sources of these transmissions could be sensor-equipped intersections or other traffic participants.

1) Measured Data: The output of the sensor fusion layer at time instance $t_{\theta}$ consists of a set of $n_{O b j}$ object hypotheses

$$
\mathbf{X}_{\theta}=\left\{X_{j}\left(t_{\theta}\right) \mid j \in\left[1, n_{O b j}\right]\right\}
$$

including the information of the ego vehicle. A single object hypothesis

$$
X_{j}\left(t_{\theta}\right)=\left\langle\mathcal{X}_{\theta}^{\Lambda_{0}}, \boldsymbol{\beta}_{\boldsymbol{o b j}}\right\rangle_{j}
$$

comprises a stochastic representation of the current motion state $\mathcal{X}_{\theta}^{\Lambda_{0}}$ and some descriptive meta data $\boldsymbol{\beta}_{\boldsymbol{o b j}} . \Lambda_{0}$ denotes here the corresponding coordinate system (CS) which could be the ego vehicle system $\Lambda_{\text {ego }}$ or the CS of a sensorequipped infrastructure $\Lambda_{x}$. In both cases, the motion state $\mathcal{X}_{\theta}$ is defined as a normal distributed random vector including the current pose $\left(x_{\theta}, y_{\theta}, \psi_{\theta}\right)^{\Lambda_{0}}$ and velocity $\left(v_{\theta}\right)$. The meta data covers the unique identifier as well as the estimated object class with dimensions (length, width and height).
2) Stored Data: In order to support the situation analysis process, the digital map is specified by a directed acyclic graph (DAG) $\mathcal{G}=\{\mathcal{R}, \mathcal{E}\}$. In this structure nodes $\mathcal{R}$ represents distinct road lane segments $(R L S)$ and the edges $\mathcal{E}$ corresponds to the connectivity between them. Similar to a object hypothesis, a road segment $j$ is defined by the tuple $\mathcal{R}_{j}=\left\langle\mathcal{Q}, \boldsymbol{\beta}_{\text {lane }}\right\rangle_{j}$, where $\mathcal{Q}$ denotes the list of stochastic way points and $\boldsymbol{\beta}_{\text {lane }}$ the appended meta data. The uncertainty of the specific points results here from the localization accuracy of the ego vehicle to the map. The meta data contains the lane width, total length, an unique identifier and the legal speed limit.

\section{B. ADAS Function}

The output of the SitA-layer comprises a formal representation of the current traffic situation and assessed maneuver hypotheses for traffic participants in the vicinity of the ego vehicle and forms the input to ADAS function layer. Generally, the objectives of this layer are to find appropriate maneuvers for the ego vehicle (maneuver planning) as well as to inform the driver and execute the planned maneuvers (driver information \& vehicle control). For autonomous driving functions, maneuver planning is of central importance (e.g. [11]).

1) Motion Planning: On a top level, the system has to make distinct decisions on the ego vehicle's desired behavior, e.g. how to obey give-way rules given an intersection's topology and the perceived objects that populate this intersection. These decisions are then translated by a motion planning algorithm into a path or trajectory (i.e. a path as a function of time). For safety functions, e.g. collision mitigation and avoidance functions, the objectives are similar. Based on the criticality assessment, maneuver planning decides whether or not to initiate emergency maneuvers (braking or steering).

2) Driver Information \& Vehicle Control: The driver is alerted based on the criticality measures derived by the SitA layer. If emergency maneuvers become necessary, these are executed via specialized vehicle controllers.

\section{THEORETICAL BACKGROUND}

\section{A. Modelling a RLS using Spline Interpolation}

To achieve a smooth and precise approximation between the consecutive way points of a road lane segment $(R L S)$ we employ cubic spline curves. A specific way point is given by $\mathbf{q}_{i}=\left[x_{i}, y_{i}, \kappa_{x, i}, \kappa_{y, 0}\right]^{T}$, where $\left(x_{i}, y_{i}\right)$ denotes the absolute position and $\left(\kappa_{x, i}, \kappa_{y, i}\right)$ the product of lane curvature $\kappa_{i}$ and normal vector $\mathbf{n}_{i}$, with $\left(\kappa_{x, i}, \kappa_{y, i}\right)^{T}=\kappa_{i} \mathbf{n}_{i}$. While the way points are available from the digital map, the $2 \mathrm{~d}$ centerline $\mathbf{s}$ of $R L S$ is interpolated piecewise as an arc length parametrized spline curve:

$$
\mathbf{s}_{i}:\left[0, l_{i}\right] \rightarrow \mathbb{R}^{2} ; \quad \mathbf{s}_{i}(l)=\mathbf{G}(l)\left(\begin{array}{c}
\mathbf{q}_{i} \\
\mathbf{q}_{i+1}
\end{array}\right) .
$$

Here, $\mathbf{G}$ denotes the matrix function of the spline, as proposed in [12]. This matrix is defined by the transitional conditions of consecutive lane segments and ensures that the spline guarantees $G^{2}$-continuity, i.e. curvature continuity. For 


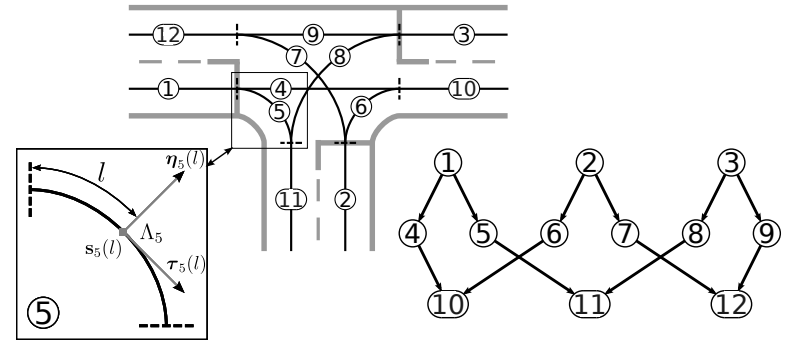

Fig. 2. Road layout comprising 12 road lane segments $(R L S)$ with corresponding DAG and exemplary local curve coordinate system (CCS)

the sake of readability, the combined vector $\left[\mathbf{q}_{i}, \mathbf{q}_{i+1}\right]^{T}$ is indicated by $\gamma_{i}$ in the remaining paper.

\section{B. Stochastic Modeling of a Curve Coordinate System}

The localization uncertainty of the ego vehicle to the digital map is accounted by a stochastic model of the way point data. To limit the computational complexity, the combined vector $\gamma_{i}$ is represented by a normal distributed random vector

$$
\gamma_{i} \sim \mathcal{N}_{8}\left(\left(\begin{array}{c}
\boldsymbol{\mu}_{i} \\
\boldsymbol{\mu}_{i+1}
\end{array}\right),\left(\begin{array}{cc}
\boldsymbol{\Sigma}_{i} & 0 \\
0 & \boldsymbol{\Sigma}_{i+1}
\end{array}\right)\right)=\mathcal{N}_{8}\left(\boldsymbol{\mu}_{i}^{*}, \boldsymbol{\Sigma}_{i}^{*}\right) .
$$

Thereby, the density function of any specific point $l_{A}$ on spline $\mathbf{s}_{i}$ in Cartesian coordinates is given by

$$
\begin{aligned}
\mathbf{s}_{i}\left(l_{A}\right) & \sim \mathcal{N}_{2}\left(\mathbf{G}\left(l_{A}\right) \boldsymbol{\mu}_{i}^{*}, \mathbf{G}\left(l_{A}\right) \boldsymbol{\Sigma}_{i}^{*} \mathbf{G}\left(l_{A}\right)^{T}\right) \\
& \sim \mathcal{N}_{2}\left(\boldsymbol{\mu}_{s}, \boldsymbol{\Sigma}_{s}\right) .
\end{aligned}
$$

In order to obtain a lane aligned coordinate system we employ the Frenet-Serret formulas for $2 \mathrm{~d}$ curves to define the tangential $\boldsymbol{\tau}_{i}$ and normal vector $\boldsymbol{\eta}_{i}$ at point $l_{A}$

$$
\begin{aligned}
\boldsymbol{\tau}_{i}\left(l_{A}\right) & \sim \mathcal{N}_{2}\left(\mathbf{G}^{\prime}\left(l_{A}\right) \boldsymbol{\mu}_{i}^{*}, \mathbf{G}^{\prime}\left(l_{A}\right) \boldsymbol{\Sigma}_{i}^{*} \mathbf{G}^{\prime}\left(l_{A}\right)^{T}\right) \\
\boldsymbol{\tau}_{\alpha} & \sim \mathcal{N}_{2}\left(\boldsymbol{\mu}_{\tau}, \boldsymbol{\Sigma}_{\tau}\right) \\
\boldsymbol{\eta}_{i}\left(l_{A}\right) & =\left(\begin{array}{cc}
0 & -1 \\
1 & 0
\end{array}\right) \boldsymbol{\tau}_{i}\left(l_{A}\right)=\mathbf{U} \mathbf{G}^{\prime}\left(l_{A}\right) \boldsymbol{\gamma}_{i} \\
\boldsymbol{\eta}_{\alpha} & \sim \mathcal{N}_{2}\left(\mathbf{U} \boldsymbol{\mu}_{\tau}, \mathbf{U} \boldsymbol{\Sigma}_{\tau} \mathbf{U}^{T}\right)=\mathcal{N}_{2}\left(\boldsymbol{\mu}_{\eta}, \boldsymbol{\Sigma}_{\eta}\right)
\end{aligned}
$$

Appending both vectors to point $\mathbf{s}\left(l_{A}\right)$ yields a stochastic model of the curve coordinate system (CCS), as shown in Fig. 2.

\section{Stochastic Coordinate Transformation}

Since $\left|\mathbf{t}_{\alpha}\right|=\left|\mathbf{n}_{\alpha}\right|=1$, the conversion of a random vector $\mathbf{v} \sim \mathcal{N}_{2}\left(\boldsymbol{\mu}_{v}, \boldsymbol{\Sigma}_{v}\right)$ defined in a origin CS $\left(\Lambda_{0}\right)$ into a stochastic $\operatorname{CCS}\left(\Lambda_{\alpha}\right)$ complies with an element-wise projection onto the coordinate axes:

$$
\mathbf{v}^{\Lambda_{\alpha}}=\mathbf{R}_{\Lambda_{\alpha}}^{\Lambda_{0}} \mathbf{v}^{\Lambda_{0}}=\left(\boldsymbol{\tau}_{\alpha} \boldsymbol{\eta}_{\alpha}\right)^{T} \mathbf{v}^{\Lambda_{0}}=\left(v_{\tau} v_{\eta}\right)^{T}
$$

Due using an auxiliary vector $\mathbf{y}=\left(\mathbf{e} \mathbf{v}^{\Lambda_{0}}\right)^{T}$ with $\mathbf{e} \in\left\{\boldsymbol{\tau}_{\alpha}, \boldsymbol{\eta}_{\alpha}\right\}$, this projection can be expressed by its quadratic form $v_{e}=\mathbf{y}^{T} \mathbf{A y}$ with $\mathbf{A}=\left(\begin{array}{cc}\mathbf{0} & \mathbf{A}^{\prime} \\ \mathbf{A}^{\prime} & \mathbf{0}\end{array}\right)$ and $\mathbf{A}^{\prime}=\left(\begin{array}{cc}1 / 2 & 0 \\ 0 & 1 / 2\end{array}\right)$.
According the principles proposed in [13] the first two moments of the resulting stochastic product $v_{e}$ is given by:

$$
\begin{aligned}
E\left\{\mathbf{y}^{T} \mathbf{A y}\right\} & =\operatorname{tr}\left[\mathbf{A} \boldsymbol{\Sigma}_{y}\right]+\boldsymbol{\mu}_{y}^{T} \mathbf{A} \boldsymbol{\mu}_{y} \\
\operatorname{Var}\left\{\mathbf{y}^{T} \mathbf{A y}\right\} & =2 \operatorname{tr}\left[\left(\mathbf{A} \boldsymbol{\Sigma}_{y}\right)^{2}\right]+4 \boldsymbol{\mu}_{y}^{T} \mathbf{A} \boldsymbol{\Sigma}_{y} \mathbf{A} \boldsymbol{\mu}_{y} .
\end{aligned}
$$

\section{Situation Model}

The main task in the situation model (SitM) is to generate a comprehensive representation of the current traffic situation and to formulate several hypotheses about the evolvement of the scene. To achieve this we propose a SitM comprising the following steps:

1) Situation Comprehension

2) Situation Prediction

The output of the SitM includes several behavioral hypotheses for each vehicle concerning the road layout and relation to other objects. This set of hypothesis will be assessed in the SitI step.

\section{A. Situation Comprehension}

One crucial aspect of successful SitA is the contextual understanding of the current traffic situation, i.e. the relationships among the traffic participants as well as to road layout or infrastructure. Here, the key lies in coupling the object hypotheses to the road network and evaluating the corresponding DAG. This leads to a probabilistic situation representation which covers a hypothetical assignment of the vehicles to all $R L S$ in its vicinity.

To classify the most probable path, on which an object vehicle is traveling, we use a probabilistic lane association. This method evaluates the likelihood of a $R L S$ concerning three features $\mathcal{F}=\left(d_{\eta}, o_{\tau}, v_{\tau}\right)$ and their respective assignment functions $g_{\{\mathcal{F}\}}$, shown in Fig. 3. Employing the conversion method mentioned on III-B, the current object state vector $\mathcal{X}_{k}^{\Lambda_{\alpha}} \sim \mathcal{N}_{4}\left(\boldsymbol{\mu}_{\alpha}, \boldsymbol{\Sigma}_{\alpha}\right)$ with expectation vector

$$
\boldsymbol{\mu}_{\alpha}=\left(\begin{array}{llll}
l_{A} & d_{\eta} & v_{\tau} & v_{\eta}
\end{array}\right)^{T} .
$$

is expressed in the CCS of $R L S_{\alpha}$. It consists of the arc length along the centerline $\left(l_{A}\right)$, the lateral deviation $\left(d_{\eta}\right)$ and the components of velocity in tangential and normal direction $\left(v_{\tau}, v_{\eta}\right)$. Using these curve-based coordinates, we determine the likelihood of all features by the product of the convolution integrals:

$$
P\left(\mathcal{F} \mid R L S_{\alpha}\right)=\prod_{i=1}^{3} \int_{-\infty}^{\infty} g_{\mathcal{F}_{i}}(\xi) p\left(\mathcal{F}_{i}-\xi\right) d \xi,
$$

where $p\left(\mathcal{F}_{i}\right) \sim \mathcal{N}_{1}\left(\mu_{\mathcal{F}}, \sigma_{\mathcal{F}}^{2}\right)$ denotes the probable density function of feature $i$ and $g_{\mathcal{F}_{i}}$ the related assessment function. Under the assumption that the prior PDF of a $R L S$ follows an uniform distribution, we assess the hypothesis "the vehicle is located in $R L S_{\alpha}$ " by utilizing the Bayes Theorem:

$$
P\left(R L S_{\alpha} \mid \mathcal{F}\right)=\frac{P\left(\mathcal{F} \mid R L S_{\alpha}\right)}{\sum_{\alpha=1}^{n_{R L S}} P\left(\mathcal{F} \mid R L S_{\alpha}\right)}
$$

The evaluated features are defined as follows: 


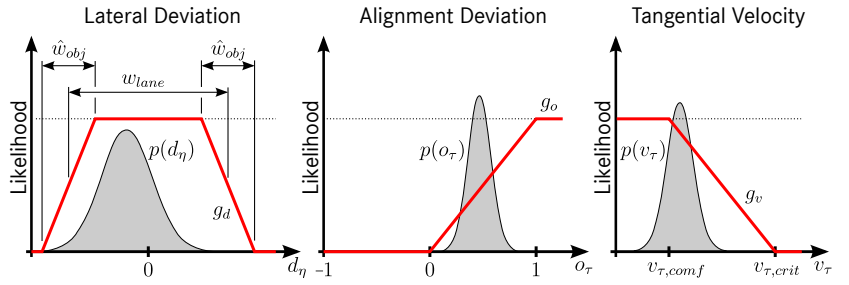

Fig. 3. Assignment function and probability density function (PDF) for extracting the likelihood of a $R L S$

1) Lateral Deviation: Obviously, the likelihood of a particular lane should be greater than zero, if a vehicle at least protrudes into it. To achieve these, the assignment functions of the lateral deviation feature $g_{d}$ is determined by the lane width $\left(w_{\text {lane }}\right)$ and the projected object width $\left(\hat{w}_{o b j}\right)$ to the normal vector of the CCS (see Fig. 3, left).

2) Alignment Deviation: In the CCS the difference between the alignment of a vehicle and the $R L S$ is evaluated by mapping the orientation of the vehicle to the tangent vector $o_{t}=\mathbf{o}^{\Lambda_{0}} \boldsymbol{\tau}_{\alpha}^{\Lambda_{0}}=v_{\tau} /|\mathbf{v}|$. Fits the vehicle orientation to the direction of the $R L S$, the corresponding assignment function becomes $g_{o}=1$. If they are aligned perpendicular or even in different directions the assignment equals zero.

3) Tangential Velocity: Previous publications regarding similar topics, e.g. [?], have shown that the velocity of a vehicle along a curved road is limited by the resulting lateral acceleration $a_{n}$. To take this into account, the assessment function of the tangential velocity $g_{v}$ is defined by two characteristically velocities. Both velocity boundaries are specified by the curvature $\kappa_{\alpha}$ at the CCS of a $R L S$ and a threshold of the lateral acceleration $\left(a_{\text {lat,comf }}, a_{\text {lat,max }}\right)$. In case of a velocity lower than the comfortable boundary $v_{t, \text { comf }}:=\sqrt{\left|a_{\text {lat }, \text { comf }}\right| / \kappa_{\alpha}}$, the assignment function equals one and decreases to zero until the velocity reaches the critical limit $v_{t, c r i t}:=\sqrt{\left|a_{\text {lat, } \max }\right| / \kappa_{\alpha}}$. Especially, when a curved $R L S$ passes away from a straight one the tangential velocity is an important feature for the lane assessment.

\section{B. Situation Prediction}

To predict the possible evolvements of the current scene, we formulate at least one motion hypothesis for each $R L S$ which was classified as relevant for an object. Depending on the road layout, a $R L S$ can offer more than one maneuver possibility which each will be covered by a separate motion hypothesis. At an urban intersection, the set of possible maneuver hypotheses $\mathbf{H}$ may consist of

$$
\begin{aligned}
\mathbf{H}= & \left\{h_{M} \mid M \in[1,4]\right\} \\
& h_{1}=\text { straight } h_{2}=\text { stop at a certain line } \\
& h_{3}=\text { left turn } \quad h_{4}=\text { right turn. }
\end{aligned}
$$

Due to the stochastic description of the vehicle states, a particular motion hypothesis comprises $K$ predicted expectation vectors $\mathbf{x}$ and covariance matrices $\boldsymbol{\Sigma}_{x}$.

For objects of type vehicle we use an Extended Kalman Filter (EKF) combined with a kinematic bicycle model $\mathbf{f}_{v e h}\left(\mathbf{x}_{k}^{\Lambda_{0}}, \mathbf{u}\right)$. This method allows us to incorporate knowledge of system dynamics and additional information from an external source (i.e. digital map). The control input vector $\mathbf{u}=\left(\delta, a_{\text {lon }}\right)^{T}$ consists of steering wheel angle resp. longitudinal acceleration.

Estimating a time-series of input values $\mathbf{U}_{k: K}$ with $T^{K}=\left\{t_{k}=t_{\theta}+k \Delta t \mid k \in\{0,1, \ldots, K\}\right\} \quad$ results in a predictive future behavior of a traffic participant. This forms a stochastic representation of a future trajectory

$$
\mathcal{X}_{k: K}^{\Lambda_{0}}=\left\{\mathcal{X}_{k} \sim \mathcal{N}_{4}\left(\mathbf{x}_{k}, \boldsymbol{\Sigma}_{x, k}\right) \mid k \in T^{K}\right\} .
$$

The arising process of behavioral prediction can be separated in a lateral and longitudinal part:

1) Lateral Behavior: To estimate the future path of a vehicle along a specified sequence of $R L S$, i.e. estimating $\boldsymbol{\delta}_{k: K}=\left\{\delta_{k} \mid k \in T^{K}\right\}$, we employ the algorithm proposed by the authors in [14]. Within the time interval $T^{K}$ the future trajectory is predicted by the EKF due to two alternating steps: The prediction step subsequently utilizes the motion model with an estimated steering wheel angle to generate the next vehicle state vector. The update step incorporates the relative properties between the predicted vehicle state and the properties of the $R L S$ in the CCS. In cases where the vehicle is not yet located entirely on the $R L S$, this algorithm ensures that the predicted trajectory successively approaches. Here, the convergence speed depends strongly on the assumed system noise which has to be learned from data.

2) Longitudinal Behavior: In order to predict the velocity profile along an estimated path, i.e. estimating $\boldsymbol{a}_{\text {lon }, k: K}=\left\{a_{\text {lon }, k} \mid k \in T^{K}\right\}$, we applied the Intelligent Driver Model (IDM) [15]. Within the IDM, the estimated future acceleration $\dot{v}$ is formulated as a time-continuous function which can be separated into two behavioral interpretations:

$$
\dot{v}=\underbrace{a\left(1-\left(\frac{v}{v_{0}}\right)^{4}\right)}_{\text {free road behavior }}-\underbrace{a\left(\frac{s_{0}+v T^{M}}{s}+\frac{v \Delta v}{2 s \sqrt{a b}}\right)^{2}}_{\text {interacting behavior }} .
$$

In the case of a free road, the interaction term is negligible. So, the calculated acceleration is determined just by the maximal allowed value $a$ as well as by the current and the desired velocity ( $v$ resp. $v_{0}$ ). The predicted velocity approaches asymptotically this target speed $v=v_{0} \sqrt[4]{1-\dot{v} / a}$.

For straight roads, the desired velocity will be a fixed value depending on the legal speed limit. However, at urban intersections the desired velocity needs to be set dynamically as normal drivers will slow down before they enter a highly curved road. To estimate the desired speed along a predicted path we limit it by the resulting lateral acceleration which is implemented in the same way as proposed in IV-A.3.

When approaching slower or standing objects, like vehicles or even traffic signs, the resulting acceleration depends additionally on the difference in position $s$ and velocity $\Delta v$. The remaining parameters denotes the minimal distance 
at still stand $s_{0}$, some safe time headway $v T^{M}$ and a comfortable deceleration value $b$.

Assuming a certain stop line in front of the vehicle $(\Delta v=v)$, a minimum kinematic deceleration $b_{k i n}:=v^{2} /(2 s)$ is necessary to avoid a collision. In such a case, the IDM acceleration reduced to

$$
\dot{v}^{i n t} \approx-\frac{v^{2}}{4 b s^{2}}=-\frac{b_{k i n}^{2}}{b}=-\beta b_{k i n},
$$

where $\beta:=b_{k i n} / b$ denotes the criticality of a predicted breaking maneuver. For $\beta>1$ the situation is characterized by an emergency stop, in contrast to a comfortable maneuver with $\beta \leq 1$.

\section{Situation Interpretation}

By determining the SitM each object hypothesis $X_{j}$ is augmented with a set of maneuver hypotheses $\mathbf{H}$. Hence, the object hypothesis $X_{j}$ is represented by the triple

$$
X_{j}\left(t_{\theta}\right)=\left\langle\mathcal{X}_{\theta}^{\Lambda_{0}}, \boldsymbol{\beta}_{\boldsymbol{o b j}}, \mathbf{H}\right\rangle_{j}
$$

where a single maneuver hypothesis $h_{M}$ is expressed by a predicted trajectory and the related likelihood of the $R L S$ $\mathbf{H}=\left\{h_{M}=\left\{\mathcal{X}_{k: K}^{\Lambda_{0}}, P(R L S)\right\} \mid M \in[1,4]\right\}$.

To infer the intention of an observed vehicle we propose in this section a behavior analysis which is based on the evidence theory, first introduced by Dempster and Shafer in 1976 [16]. The main benefit of this theory is the ability to consider the imprecision of a situation due to the lack of knowledge in addition to the empirical uncertainty of the perception. With the key principals of the DempsterShafer theory of evidence (DST), described in the following, we evaluate the provided maneuver hypotheses by a multi criteria assessment.

\section{A. Dempster-Shafer Theory of Evidence}

In DST, a finite universal set $\Omega=\left\{h_{i}\right\}$ of elementary (singleton) hypotheses $h_{i}$ is called frame of discernment. The set of all subsets of $\Omega$ is its power set $2^{\Omega}$. It is required that all hypothesis have to be unique, not overlapping and mutually exclusive. Any subset $A$ of those $2^{\Omega}$ sets may consist of a single hypothesis or of a conjunction of hypotheses.

A special feature of DST is the possibility to compute a lower and an upper bound (belief and plausibility) of the precise probability of set $A$. For this purpose the belief functions theory includes three basic functions for mapping the power set $2^{\Omega}$ to the interval $[0,1]$.

The basic belief assignment (BBA) $m$ assigns an evidential weight to a set $A$ and fulfills

$$
m: 2^{\Omega} \rightarrow[0,1] ; \quad \sum_{A \subseteq \Omega} m(A)=1 ; \quad m(\emptyset)=0 .
$$

Each $A$ that holds $m(A)>0$ is called a focal element. When $\Omega$ is the only focal element, the BBA is called the void BBA. This can be interpreted as complete ignorance.

With the definition of the BBA the degree of Belief (bel) describes the support for the proposition, i.e. the minimal

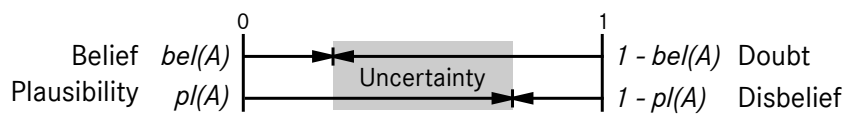

Fig. 4. Uncertainty, belief, plausibility and its complements of a BBA

belief to set $A$. This lower bound is defined as the sum of all BBA concerning the set of interest:

$$
\text { bel }: 2^{\Omega} \rightarrow[0,1] ; \quad \operatorname{bel}(A):=\sum_{B \subseteq A ; B \neq \emptyset} m(B) .
$$

The upper bound of the probability, the degree of Plausibility $(p l)$, commits the maximal belief that can be transferred to $A$. It is defined by the sum of all BBAs that intersect the set of interest:

$$
p l: 2^{\Omega} \rightarrow[0,1] ; \quad p l(A):=\sum_{B \cap A \neq \emptyset} m(B) .
$$

A graphical representation of these basic functions is shown in Fig. 4. The gap between the belief and plausibility describes the evidential interval range which represents the uncertainty concerning the set A.

In the case an evidential source has only a certain level of trust, this can be taken into account as probability $\rho$. As proposed in [7], this probability can be used to discount a $\mathrm{BBA}$ prior to combination with another BBA and is defined as:

$$
m^{\rho}(A)=\left\{\begin{array}{ll}
\rho m(A), & A \neq \emptyset \\
\rho m(\Omega)+1-\rho, & A=\Omega
\end{array} .\right.
$$

The DST and its extensions also provide several methods to combine different BBA's which we distinguish into two cases of application.

To combine the basic belief assignments from $n$ independent sources at a particular time instance we use the Dempster's rule of combination. It is called the orthogonal sum and is donated by

$$
m_{1 \oplus 2 \oplus \cdots \oplus n}=m_{1} \oplus m_{2} \oplus \cdots \oplus m_{n}
$$

which is a commutative and associative operation but not idempotent or continuous.

In the case of $A \in 2^{\Omega}$, the aggregation of two BBA's $m_{1}$ and $m_{2}$ can be calculated in the following manner:

$$
\begin{aligned}
m_{1 \oplus 2}(\emptyset) & =0, \\
m_{1 \oplus 2}(A) & =\frac{\sum_{B \cap C=A} m_{1}(B) m_{2}(C)}{1-K} .
\end{aligned}
$$

Where the coefficient $K=\sum_{B \cap C=\emptyset} m_{1}(B) m_{2}(C)$ represents the existing conflict aspect between the combined sources. When $K=1$, this operation will yield counterintuitive results.

In [17] Yager developed a modified dempster rule, which does not change the evidence through the normalization coefficient $(1-K)$. Potential conflicts will be allocated to the universal set $(\Omega)$ instead to the null set $(\emptyset)$. In this way, the conflict in $K$ is interpreted as the degree of ignorance. This rule is used especially in the case of updating an 


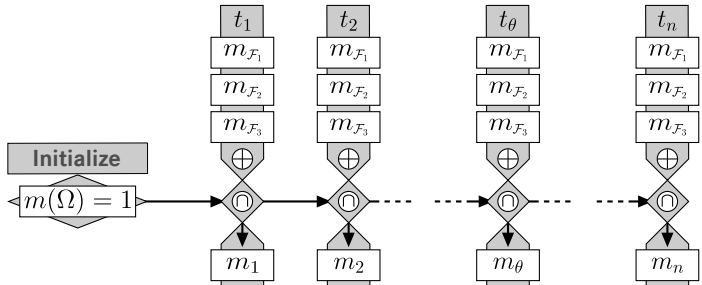

Fig. 5. Strategy for combining the determined basic belief assignments (BBA) of individual features. The BBAs at a single time slice are combined using the Dempster rule (22). The combination of the resulting BBAs at consecutive time steps is realized by the Yager rule (23).

already combined structure when new information becomes available. Hence, we employ it to combine consecutive time slices. The modified Dempster's rule of combining is defined as follows:

$$
\begin{aligned}
& m_{1 \bigcirc 2}(\emptyset)=0, \\
& m_{1 \bigcirc 2}(A)=\sum_{B \cap C=A} m_{1}(B) m_{2}(C), \\
& m_{1 \bigcirc 2}(\Omega)=\sum_{B \cap C=\Omega} m_{1}(B) m_{2}(C)+K
\end{aligned}
$$

In order to facilitate decision making on BBA's, we use the pignistic probability transformation (PPT) [18]:

$$
\operatorname{bet} P(A)=\sum_{B \subseteq \Omega} \frac{|A \cap B|}{|B|} m(B),
$$

where $|\cdot|$ denotes the number of elementary hypotheses in $\cdot$ The PPT links the evidence theory (represented on $2^{\Omega}$ ) to the probability theory (on $\Omega$ ) and is limited by the results of belief and plausibility function: $\operatorname{bel}(A) \leq \operatorname{bet} P(A) \leq p l(A)$.

\section{B. Hypothesis Assessment}

To classify the intended maneuver of a vehicle at an intersection, we assess the formulated maneuver hypotheses by combining the evidence of multiple BBAs.

The applied combination strategy, represented in Fig. 5, distinguishes between the combination of a single time step using the Dempster Rule (22) and the consecutive fusion of the resulting BBAs employing the Yager-Rule (23). The regarded frame of discernment complies the set of maneuver possibility of a vehicle. Therefore each BBA is established by a single feature concerning the predicted trajectories or contextual information.

1) BBA of Prediction Accuracy: Estimating future trajectories from a current state vector results in a strong correlation of the predicted position and velocity. This is the reason why we rate the accuracy of a behavior hypothesis by evaluating the predicted velocity profile $\hat{v}_{\tau}(l)$ along the estimated path in coordinates of CCS. An appropriate method to assess the prediction error of hypothesis $h_{M}$ offers the following distance measure

$$
d_{\mathcal{F}_{1}}\left(l_{A}, v_{\tau}, \hat{v}_{\tau}^{h_{M}}\right)=\frac{\left|v_{\tau, t}\left(l_{A}\right)-\hat{v}_{\tau}\left(l_{A}\right)\right|}{\frac{1}{n-1} \sum_{t=2}^{\theta}\left|v_{\tau, t}-v_{\tau, t-1}\right|},
$$

which evaluates the difference between the actual and the predicted tangential velocity from the previous time step
$\left(v_{t}\left(l_{A}\right)\right.$ and $\hat{v}_{\tau}\left(l_{A}\right)$, respectively) at current arc length $l_{A}$. Additionally it is scaled by the average of the actual change in the velocity of the vehicle. Due to the effect of the denominator, the resulting distance measure $d_{\mathcal{F}_{1}}$ depends on the dynamic of the vehicle or the noise of the measurement. In this way, the metric penalizes a prediction error less if the actual velocity strongly varies or more if not.

Note that the applied metric is similar to the Mean Absolute Scaled Error (MASE, see [19]), except the last averaging over time. This part is neglected, since the DST is used to consider the time evolution of the error.

Following Eqn. (17), the evidential weight of a feature $\mathcal{F}$ needs to be limited to $[0,1]$. Thus, the distance measure has to mapped on this interval which forms the first BBA:

$$
m_{\mathcal{F}_{1}}=\bigoplus\left\{m_{\mathcal{F}_{1}}^{\rho}\left(h_{M}\right)=e^{-\lambda d_{\mathcal{F}_{1}}} \mid h_{M} \in \mathbf{H}\right\}
$$

discounted by the likelihood of $R L S(\rho:=P(R L S))$, which the hypothesis $h_{M}$ starts from. Additionally, the parameter $\lambda$ controls the sensitivity of the feature depending on the distance measure. For $\lambda \approx 2$ we obtained the best trade-off between sensitivity and robustness.

2) BBA of Maneuver Criticality: In case of a brake maneuver the predicted velocity must decrease along the estimated path. This holds true also for turn maneuvers on highly curved roads, if the current velocity is too fast for a comfortable lateral acceleration. As defined in section IVB.2, the criticality of such a maneuver can be determined by the parameter $\beta:=b_{k i n} / \hat{b}$.

For a left or right turn, $\hat{b}$ denotes the maximum deceleration $\dot{v}$, estimated by Eq. (14). Predicting a brake maneuver at a certain stop line the parameter $\hat{b}$ is set to the so-called maximum comfortable deceleration $\hat{b}_{\text {comf }}$, which can be defined by the user.

The feature associated with this maneuver criticality $\beta$ is formulated as the rejection of the considered hypothesis:

$$
m_{\mathcal{F}_{2}}:= \begin{cases}m_{\mathcal{F}_{2}}^{\rho}\left(\Omega \backslash h_{M}\right)=1, & \text { if } \beta>1 \\ m_{\mathcal{F}_{2}}^{\rho}(\Omega)=1, & \text { otherwise }\end{cases}
$$

3) BBA of Velocity Criteria: In the context of urban intersections, the upper limit of the predicted velocity profile is defined by the going straight hypothesis. Accordingly, the lower limit of the profile corresponds to the predicted velocity of the braking maneuver. In such cases, where the observed vehicle is accelerating stronger as predicted, the measured value of the actual velocity is higher or lower as these borders. This can be interpreted as confirmation for the going straight or braking maneuver. The BBA of this feature is formulated as follows:

$$
m_{\mathcal{F}_{3}}:= \begin{cases}m_{\mathcal{F}_{3}}^{\rho}\left(h_{\text {straight }}\right)=1, & \text { if } v_{\tau} \geq \hat{v}_{\tau}^{h_{\text {straight }}} \\ m_{\mathcal{F}_{3}}^{\rho}\left(h_{\text {brake }}\right)=1, & \text { if } v_{\tau} \leq \hat{v}_{\tau}^{h_{\text {brake }}} \\ m_{\mathcal{F}_{3}}^{\rho}(\Omega)=1, & \text { otherwise }\end{cases}
$$

Further descriptions of semantic features to formulate additional BBAs can be found in [1]. 


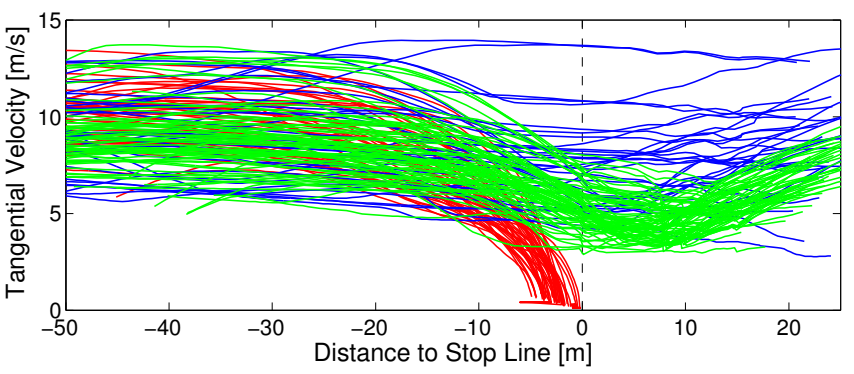

Fig. 6. Velocity profiles in tangential direction of the corresponding RLS for going straight (blue), braking (red) and turning left / right maneuver (green). The position of the particular stop line is indicated by dashed line.

\section{Evaluation}

To evaluate the presented approach, we analyze 157 maneuver recorded at an intersection in Ulm, Germany. Fig. 2 shows the layout of this intersection with the corresponding DAG. The maneuvers are performed by 5 different drivers by approaching from all three sides and includes 33 going straight, 52 braking and 72 turning maneuvers. The velocity profiles along the tangential direction of a corresponding $R L S$ are given in Fig. 6. In order to achieve comparability between the recorded trajectories, each maneuver is determined in relation to the distance to the stop line of the particular side.

\section{A. Assessing the Maneuver Hypotheses}

To determine the classification power of our approach, four exemplary maneuvers are discussed in the following, illustrated in Fig. 7, 8 and 9. Within the upper graphs of these figures the black line displays the observed velocity profile of the vehicle. The predicted trajectories regarding the different behavior hypotheses are indicated by the dotted lines. As in Fig. 6, the colors refers to the predicted maneuver; blue for going straight, red for braking and green (resp. magenta) for turning maneuvers. The lower graphs show the corresponding beliefs to the individual hypothesis. Here, the thick colored lines denotes the pignistic probability (c.f. 24), which are limited by the belief and probability values (transparent area).

1) Driving Straight: In the going straight example, shown in Fig. 7, the object vehicle approaches the intersecting from $R L S 3$ and leaves it from $R L S 12$. Up to a distance of $20 \mathrm{~m}$ to the stop line, the vehicle slows moderately and the belief in the turning hypothesis dominates. At a distance less then $20 \mathrm{~m}$, the vehicle is too fast for a comfortable turning maneuver and the belief in the straight maneuver is increasing. Since the other maneuvers are still possible the uncertainty about the actual maneuver remains big in these area. Concurrently, the braking maneuver becomes more plausible. But because no BBA is directly supporting it, the belief in this maneuver equals zero.

2) Braking: Fig. 8 represents a braking maneuver example where the vehicle is approaching on $R L S 1$. Before the vehicle stops to a halt, the velocity slows less in the range of 20 to $10 \mathrm{~m}$ in front of the stop line. As a consequence,
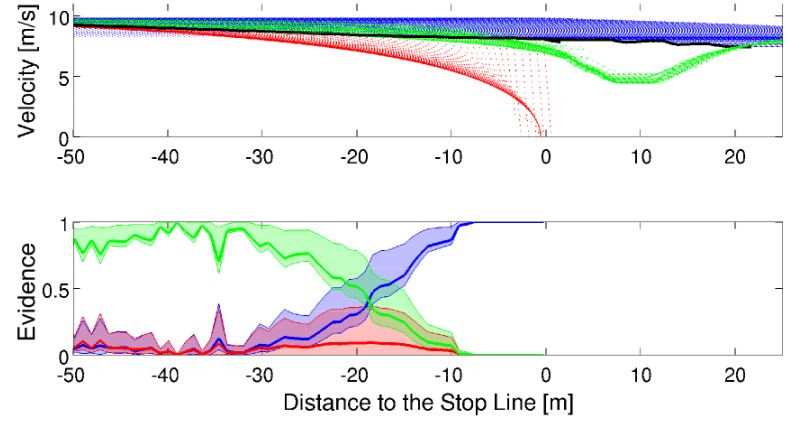

Fig. 7. Driving Straight: vehicle was approaching the intersection on $R L S 3$ and was leaving it on $R L S 12$

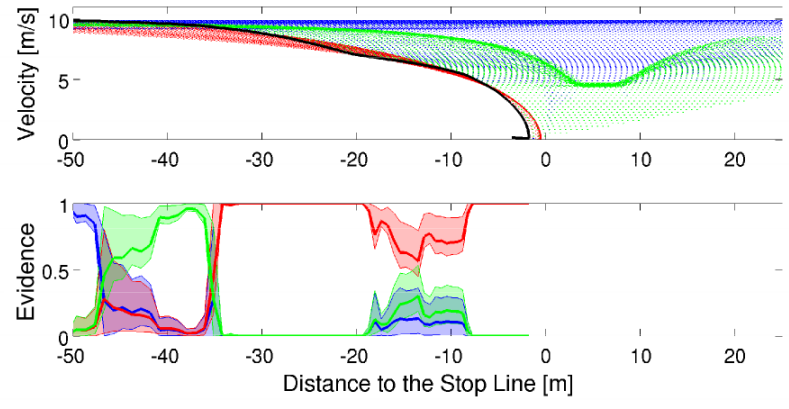

Fig. 8. Braking: vehicle stops in front of the stop line of $R L S 1$

the belief in the braking maneuver becomes less and the plausibility of the other behavior hypotheses increases.

3) Turning: Two representative examples of turn maneuvers are shown in Fig. 9. In the first example the vehicle approaches the intersection with almost constant velocity, so the belief switches between going straight and turning maneuver. At a distance of $15 \mathrm{~m}$, the vehicle slows to the predicted turn velocity and the plausibility of this hypothesis becomes 1 . Since all other hypotheses are still possible, the belief in making a turn maneuver is less than 0.7. In the second example the road layout offers only two alternative turning maneuver, one to the left (green) and one to the right (magenta). Since both velocity profiles are quite similar, the probability of both hypotheses are similar as well and the obtained results of the DST are very uncertain. This holds true until a distance of about $2 m$ to the stop line, when the turning right hypothesis becomes true.

\section{B. Classification Results}

The classification performance regarding the set of recorded maneuvers is evaluated by the Area Under the Curve (AUC) of the Receiver Operating Characteristic (ROC) [20]. Here, the ROC curve is determined by evaluating the pignistic probability of the DST regarding the three behavioral hypothesis in an one class vs. all other classes manner. Figure 10 displays how the AUC of each ROC improves when the observed vehicle gets closer to the reference line. Note that beside the classification power the main advantage of the DST lies in the ability to model the imprecision of a situation by the distance between belief and plausibility. 

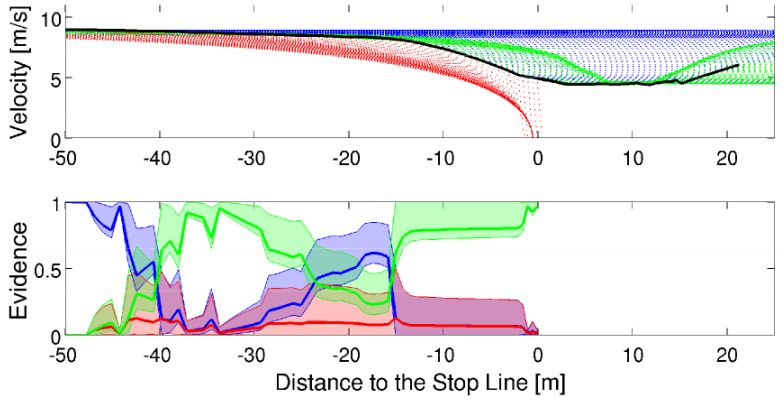

(a) Turning Left: vehicle approaches on $R L S 3$ and turns off to the left on $R L S 11$
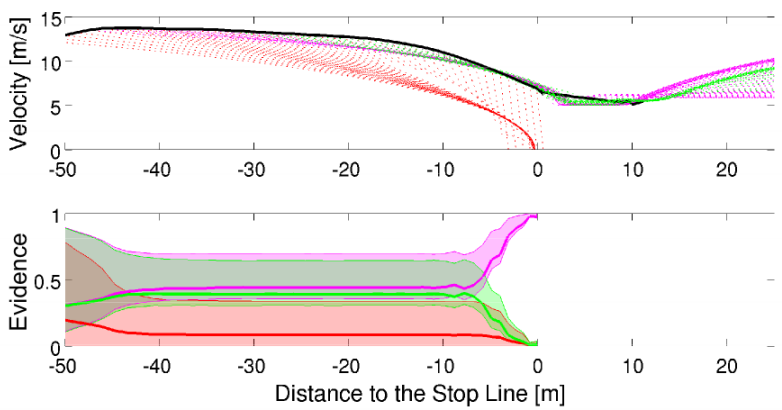

(b) Turning Right: Approaching from $R L S 2$, the road layout offers two maneuvers, one to the left (green) and one to the right (magenta)

Fig. 9. Turning: two examples of a turning maneuver

\section{CONCLUSION}

In this paper, a new situation analysis concept has been proposed which treats the two categories of the uncertainties in a traffic situation separately. This includes a associative and predictive situation model (SitM), which provides a contextual description of the current traffic scene as well as a probabilistic prediction method to formulate various hypotheses regarding the evolvement of the situation. Furthermore, we introduced an evidence based situation interpretation (SitI) to assess the several behavior hypotheses as well as to determine the inherent uncertainty. Especially in ambiguous situations, this concept provides the ADAS function more information for appropriate reactions to the scene. This is achieved by the ability to model the imprecision of a situation by the distance between belief and plausibility. The capability in behavior recognition of the concept was demonstrated by evaluating of 157 maneuvers at an urban intersection. Beside map data, the proposed concept is not relying on training data and so it is not limited to previous known traffic scenarios.

Future work will focus on interactions between a highly automated vehicle and traffic participants in its vicinity. Especially on narrow roads with oncoming traffic or at the entrance to a roundabout interacting behavior is essential for a smooth traffic flow.

\section{REFERENCES}

[1] A. Hermann and J. Desel, "Driving situation analysis in automotive environment," 2008 IEEE International Conference on Vehicular Electronics and Safety, pp. 216-221, Sept. 2008.
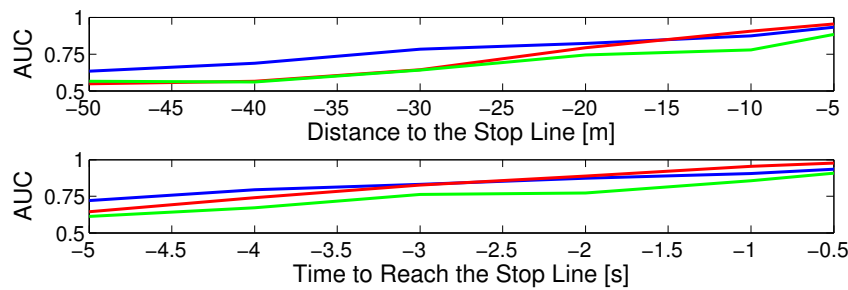

(a) Area under the ROC curve based on the classification performance of the DST depending on the distance to stop line resp. the time interval the vehicle requires to reach the stop line.
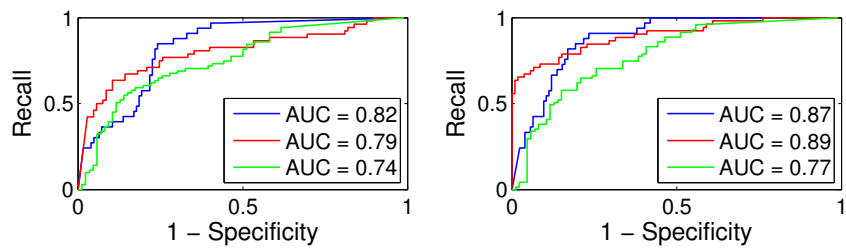

(b) ROC Curves evaluated at a distance of $20 m$ to the stop line (left) resp. at a time-to-reach the stop line of $2 s$ (right)

Fig. 10. Classification results of the DST. The colored lines represent the assessed behavioral hypotheses $($ blue $=$ straight, red $=$ brake, green $=$ turn $)$

[2] A. Tamke, T. Dang, and G. Breuel, "A flexible method for criticality assessment in driver assistance systems," 2011 IEEE Intelligent Vehicles Symposium (IV), no. Iv, pp. 697-702, June 2011.

[3] A. Jousselme, P. Maupin, and E. Bossé, "Uncertainty in a situation analysis perspective," International Conference on Information Fusion, pp. 1207-1214, 2003.

[4] M. Tsogas, G. Thomaidis, P. Lytrivis, and A. Amditis, "Detection of maneuvers using evidence theory," 2008 IEEE Intelligent Vehicles Symposium, pp. 126-131, June 2008.

[5] J. Roy, "Towards multiple hypothesis situation analysis," Information Fusion, 2007

[6] T. Gindele, S. Brechtel, and I. S. Laboratories, "Learning Context Sensitive Behavior Models from Observations for Predicting Traffic Situations," no. Itsc, pp. 1764-1771, 2013.

[7] D. Meissner, S. Reuter, B. Wilking, and K. Dietmayer, "Road User Tracking Using a Dempster-Shafer Based Classifying Multiple-Model PHD Filter," pp. 1236-1242, 2013.

[8] G. S. Aoude, V. R. Desaraju, L. H. Stephens, and J. P. How, "Driver Behavior Classification at Intersections and Validation on Large Naturalistic Data Set," vol. 13, no. 2, pp. 724-736, 2012.

[9] J. Roy, D. Support, S. Section, and E. Valcartier, "From Data Fusion to Situation Analysis."

[10] "Ko-FAS - Ko-PER - Cooperative Perception." [Online]. Available: http://ko-fas.de/english/ko-per-cooperative-perception.html

[11] J. Ziegler, P. Bender, T. Dang, and C. Stiller, "Trajectory Planning for Bertha," Intelligent Vehicles Symposium, 2014.

[12] C. Hasberg, Simultane Lokalisierung und Kartierung spurgeführter Systeme. Vol. 19. KIT Scientific Publishing, 2011.

[13] S. R. Searle, Linear Models. John Wiley \& Sons, 1971.

[14] D. Petrich, T. Dang, D. Kasper, G. Breuel, and C. Stiller, "Map-based long term motion prediction for vehicles in traffic environments," in 16th International IEEE Conference on Intelligent Transportation Systems (ITSC 2013). IEEE, Oct. 2013, pp. 2166-2172.

[15] M. Treiber, A. Hennecke, and D. Helbing, "Congested traffic states in empirical observations and microscopic simulations," Physical Review E, 2000.

[16] R. U. Kay, "Fundamentals of the Dempster-Shafer theory and its applications to system safety and reliability modelling," 2007.

[17] R. Yager, "On the Dempster-Shafer framework and new combination rules," Information sciences, vol. 137, pp. 93-137, 1987.

[18] P. Smets, "THE COMBINATION OF EVIDENCE IN THE TRANSFERABLE BELIEF MODEL." pp. 1-36.

[19] R. J. Hyndman, "Another look at forecast-accuracy metrics for intermittent demand," no. 4, pp. 43-46, 2006.

[20] T. Fawcett, "An introduction to ROC analysis," Pattern Recognition Letters, vol. 27, no. 8, pp. 861-874, June 2006. 